\title{
Orientation Control of Biological Cells Under Inverted Microscopy
}

\author{
Xinyu Liu, Member, IEEE, Zhe Lu, Member, IEEE, and Yu Sun, Senior Member, IEEE
}

\begin{abstract}
Orientation control of biological cells under inverted microscopes is important for cell birefringent imaging and micromanipulation. Taking our microrobotic mouse embryo injection research as an example, this paper presents a cell orientation control system operated under inverted microscopes. A compact motorized rotational stage for inverted microscopy was developed for orienting the polar body of mouse embryos away from the injection site to avoid damage of cellular organelles. An in-house developed microdevice was used for immobilizing many cells into a regular pattern. The polar body is tracked by a visual tracking algorithm with a translation-rotation-scaling motion model, providing image position feedback to an image-based visual servo controller that is responsible for online calibration of coordinate transformation during visually servoed orientation of the first embryo. High-speed, automatic cell orientation is then conducted on other embryos in the same batch of immobilized embryos through coordinate transformation and 3-DOF closed-loop position control. Experimental results demonstrate that the cell-orientation system is capable of orienting mouse embryos at a high speed of $720 \%$ with an accuracy of $0.24^{\circ}$.
\end{abstract}

Index Terms-Cell manipulation, cell orientation, inverted microscopy, microrobotics, rotational stage, visual servo control.

\section{INTRODUCTION}

$\mathbf{R}$ OBOTIC manipulation of microobjects [1] has important applications in microdevice prototyping [2]-[4] and cell biology [5]. Inverted microscopes are the most common platforms for imaging biological samples and for the manipulation of cells. When a cell is imaged for birefringent features that are orientation dependent [6], [7] or when a cell is manipulated [8], [9], the cell needs to be oriented properly. Existing motion stages [10] for inverted microscopy use are limited to producing $X-Y$ translational motions. This paper presents 1) the first rotational stage that operates together with a motorized $X-Y$

Manuscript received January 31, 2010; revised June 17, 2010; accepted June 18, 2010. Date of publication August 9, 2010; date of current version August 30 , 2011. Recommended by Technical Editor S. Fatikow. This work was supported in part by the Natural Sciences and Engineering Research Council of Canada and in part by the Ontario Ministry of Research and Innovation. The work of Y. Sun was supported by the Canadian Research Chairs program. The work of $X$. Y. Liu was supported by the Ontario Graduate Scholarship program.

X. Y. Liu was with the Advanced Micro and Nanosystems Laboratory, Mechanical and Industrial Engineering Department, University of Toronto, Toronto, ON M5S 3G8, Canada. He is now with the Department of Chemistry and Chemical Biology, Harvard University, Cambridge, MA 02138 USA (e-mail: xliu@gmwgroup.harvard.edu).

Z. $\mathrm{Lu}$ and Y. Sun are with the Advanced Micro and Nanosystems Laboratory, Mechanical and Industrial Engineering Department, University of Toronto, Toronto, ON M5S 3G8, Canada (e-mail: zhe.lu@utoronto.ca; sun@mie.utoronto.ca).

Color versions of one or more of the figures in this paper are available online at http://ieeexplore.ieee.org.

Digital Object Identifier 10.1109/TMECH.2010.2056380

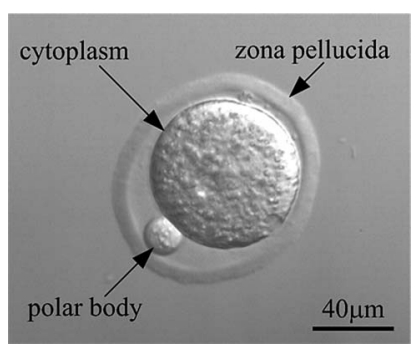

Fig. 1. Polar body of a mouse embryo must be properly oriented to avoid micropipette penetration.

stage for inverted microscopes and 2) control strategies for controlling the orientation of a biological sample. The technologies are described in the scenario of our microrobotic cell injection research; however, the system and control strategies are widely applicable to other cell imaging and manipulation tasks [11].

In microrobotic injection of mouse oocytes and embryos, the polar body of the cell (see Fig. 1) must be positioned away from the penetration site to avoid polar body damages and increase the chance of further cellular development. In the state-of-theart manual and microrobotic mouse embryo injection [12]-[16], cell orientation is achieved with a holding pipette by repeated vacuum sucking and releasing until the polar body is rotated away from the penetration site. Due to poor controllability, orienting embryos is a slow and trial-and-error process [9]. Furthermore, the use of a holding pipette makes switching from one embryo to another highly time consuming. These limitations prevent the realization of fully automated, high-speed microrobotic injection.

This paper presents a system for automated cell orientation control. The system employs a microfabricated glass device to immobilize many mouse embryos into a regular pattern. As the schematic shows in Fig. 2(a), evenly spaced micrometer-sized through-holes $(37 \mu \mathrm{m})$ immobilize individual mouse embryos $(\sim 100 \mu \mathrm{m})$ by applying a negative pressure through the bottom channel. Fig. 2(b) shows an array of immobilized cells. Although this approach permits parallel immobilization of many cells, cell orientations are largely random [see Fig. 2(b)], requiring each embryo to be properly oriented before automated microrobotic injection is performed. We previously reported device design and microfabrication in [17]. This paper reports the motion control system and orientation control approaches.

To realize automated cell orientation, a compact motorized rotational microscopy stage, which is commercially unavailable, was developed. A position-vision-based system was employed for automated cell orientation control. The polar body was tracked using a visual tracking algorithm to provide visual 

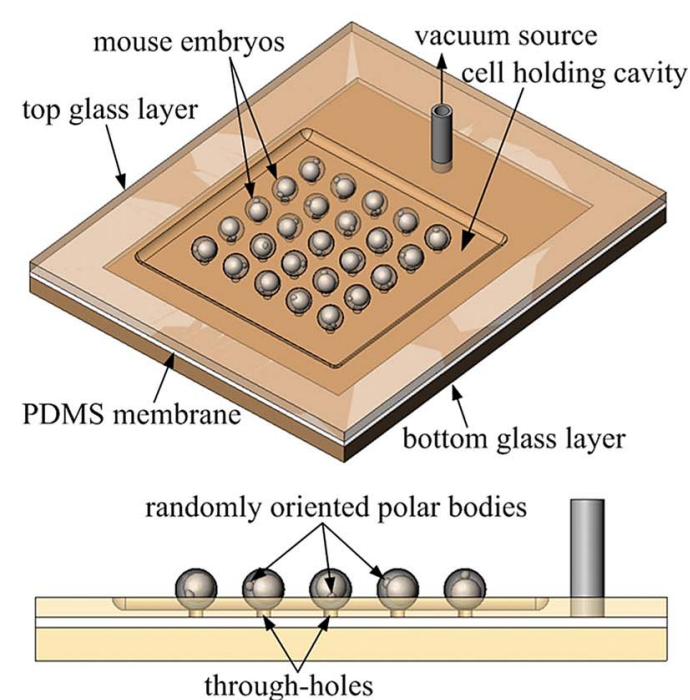

(a)

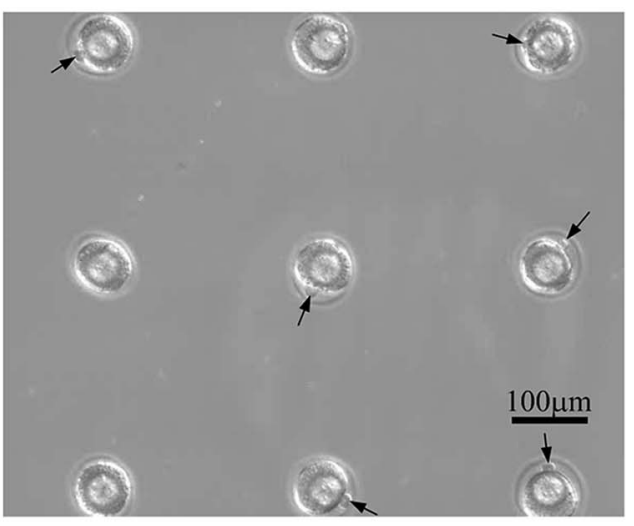

(b)

Fig. 2. (a) Schematic of microdevice for immobilizing mouse embryos. (b) $3 \times 3$ array of immobilized cells where polar bodies (labeled by arrows) are randomly oriented.

feedback. The transformation between the rotational stage coordinate frame and the $X-Y$ stage coordinate frame is calibrated during image-based visual servo control. The polar body of a cell is oriented away from the injection site through coordinate transformation and 3-DOF closed-loop position control. Experimental results demonstrate that the cell orientation system is capable of precisely orienting mouse embryos at a maximum speed of $720^{\circ}$ s with an accuracy of $0.24^{\circ}$.

\section{Rotational Stage Design}

Considering inverted microscopy characteristics and microrobotic system integration requirements, the cell orientation stage must: 1) be able to produce smooth and fast rotational motion; 2) not block the light path; 3) possess a sample clamping mechanism to make the cell sample close enough to the microscope objectives and, therefore, within the working distance; and 4) have a compact structure to permit the rotational stage to be readily mounted onto a commonly used $X-Y$ mi- (a)
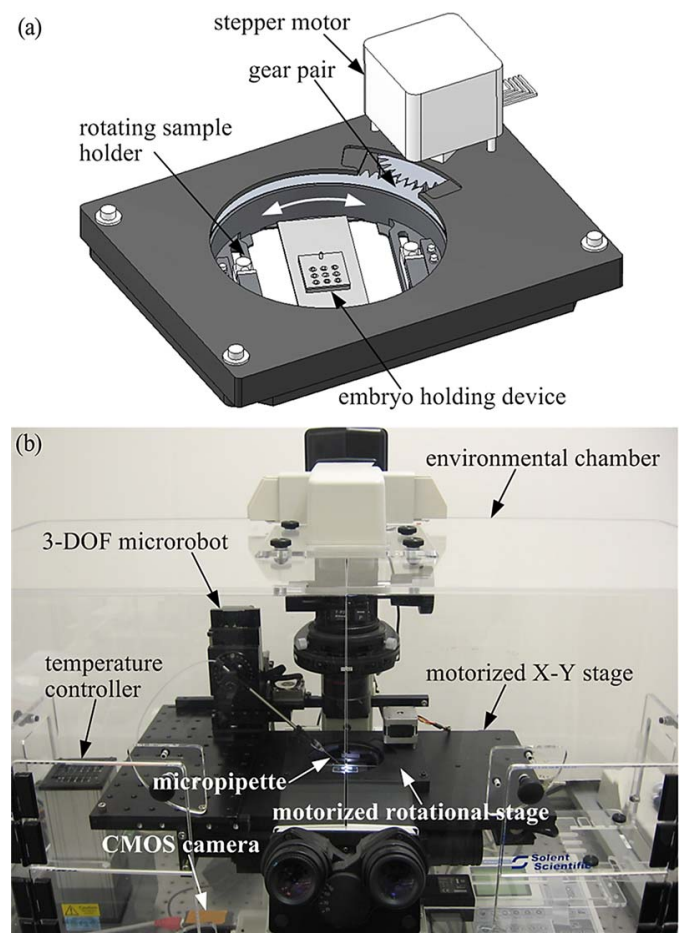

Fig. 3. (a) 3-D model of the motorized rotational microscopy stage. (b) Microrobotic cell injection system setup.

croscopy stage. As no commercially available rotational stages meet these requirements, a rotational stage was developed to enable automatic cell orientation control.

Fig. 3(a) shows a 3-D model of the rotational stage that includes a stepper motor, a pair of spur gears (gear ratio: 2.78:1) for transmitting rotational motions, and a rotating sample holder with a sliding clamp that is capable of holding glass slides of different sizes. The stage does not introduce any obstruction into the optical path for cell observation. The choice of the gear pair provides more precise motion transmissions, smoother rotational motions, and more compact structures over other motion transmission mechanisms (e.g., belt-pulley). The sliding clamp is located on the very bottom of the rotating sample holder to fix a sample within the working distances of the microscope objectives. The rotational stage is capable of orienting samples with a positioning resolution of $0.08^{\circ}$ and a maximum rotational speed of $1800^{\circ} / \mathrm{s}$.

\section{MOUSE EMBRYO INJECTION SYSTEM}

The microrobotic mouse embryo injection system [see Fig. 3(b)] consists of an inverted microscope (TE-2000S, Nikon) with a CMOS camera (A601f, Basler), the in-house developed rotational stage and mouse embryo holding device, a motorized $X-Y$ translational stage (ProScan II, Prior), a 3-DOF microrobot (MP-285, Sutter) for controlling the injection pipette $\left(45^{\circ}\right.$ tilting angle) to diagonally penetrate cells, a host computer $(3.2 \mathrm{GHz}$ CPU, 1 GB RAM) with a motion control board (PCIe-6259, National Instruments), and a temperature-controlled chamber (Solent Scientific) to maintain cells at $37^{\circ} \mathrm{C}$. 
(a)

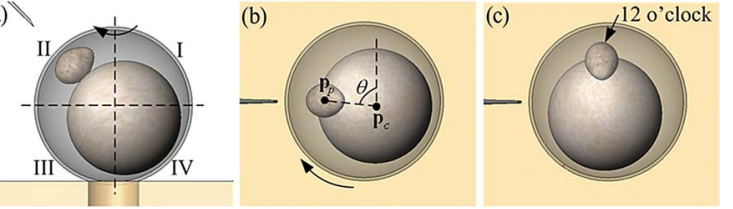

Fig. 4. Mouse embryo orientation. (a) Side view and (b) top view of the embryo and injection micropipette before orientation. (c) Top view of the embryo after orientation control. Polar body is now at 12 o'clock position. Micropipette injects a cell at $45^{\circ}$

\section{SPECIFying InITIAL POLAR Body LOCATION}

The image processing algorithms [18] developed in this study for recognizing cell structures (zona pellucida, cytoplasm, and polar body as shown in Fig. 1) have a success rate of approximately $70 \%$ because size and shape of the polar body can vary significantly across cells, and some cells do not contain very visible polar bodies. Therefore, the system presently accepts computer mouse clicking inputs from a user for reliably obtaining the initial location of the polar body. A human operator uses a total of two times computer mouse clicking on the cytoplasm center [see $\mathbf{p}_{c}$ in Fig. 4(b)] and the polar body center [see $\mathbf{p}_{p}$ in Fig. 4(b)] to specify $\mathbf{p}_{c}$ and $\mathbf{p}_{p}$ to the system in the graphical user interface of the control software.

When the polar body of an embryo is within the depth of the field, the human operator directly selects image coordinates of the cytoplasm and polar body centers. When the polar body is not visible (out of focus), the embryo is focally scanned by the $z$-motor on the microscope. During this scanning process, the human operator identifies and selects the polar-body center. The control software accepts the image coordinates from user input and the vertical coordinate of the polar-body center from the encoder feedback of the $z$-motor on the microscope.

This minimal human input is retained to guarantee system reliability; however, subsequent feature tracking and control procedures for orienting the cell are automatically conducted by the system without the need of human intervention.

\section{Cell Orientation Control}

Before the micropipette penetrates an embryo, the embryo must be properly oriented. When the polar body is present in the space of quadrant II in Fig. 4(a), there are risks of either direct polar body penetration or large-stress-induced polar body damage. The desired target orientation is either 12 o'clock or 6 o'clock [see Fig. 4(c)]. When the polar body is located in other quadrants or the polar body is exactly along the vertical axis, no orientation control is conducted.

\section{A. Overall Control Sequence}

Fig. 5 shows the overall sequence of cell orientation control. Since no embryo is coincident along the rotational axis of the rotational stage (see Fig. 6), coupled translational motions during rotation cause the embryo to move beyond the field of view. For the first embryo in a cell batch, the $X-Y$ stage is controlled via image-based visual servoing (see Fig. 5 left column, Section VB, and Fig. 7) to always keep the embryo in the field of view

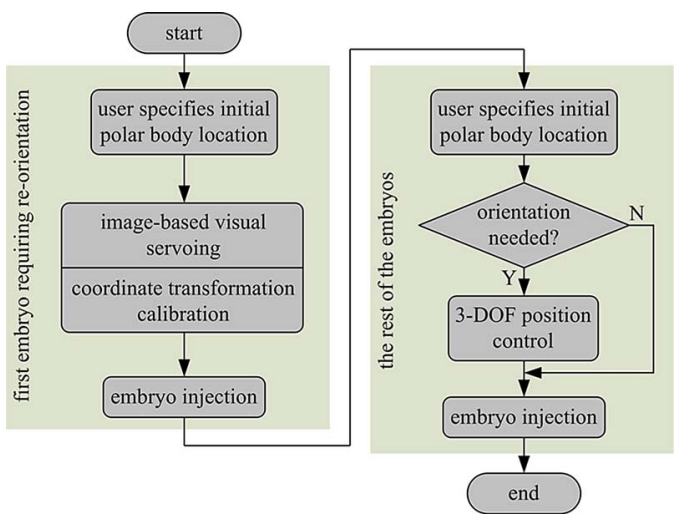

Fig. 5. Overall sequence of the cell orientation control.

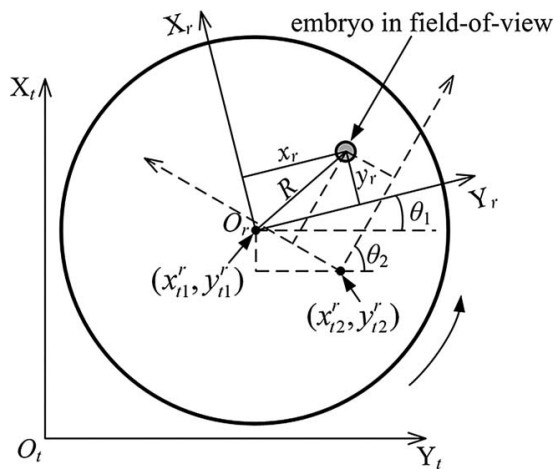

Fig. 6. Calibration of coordinate transformation

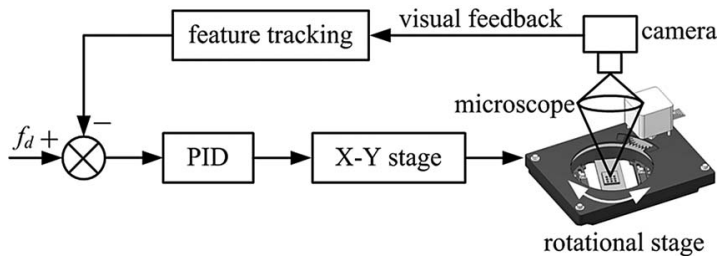

Fig. 7. Image-based visual servoing of the $X-Y$ translational stage.

during orientation. Visual servoed orientation of the first embryo enables online calibration of coordinate transformation between the rotational stage frame and the $X-Y$ translational stage frame, which is the kinematic basis of the following closed-loop position control. For the rest of the embryos in the batch (see Fig. 5, right column), closed-loop position control is employed for controlling the $X-Y$ translational stage to bring the embryo back into the field of view, permitting high-speed cell orientation (see Section V-C and Fig. 8).

Although the slow dynamic responses of visual servoing due to low vision sampling frequencies $(30 \mathrm{~Hz})$ result in a low speed for cell orientation, this calibration procedure is only required/conducted on the first embryo for an entire batch of embryos immobilized on the embryo holding device since pitches $(300 \mu \mathrm{m})$ between adjacent embryos are accurately known from microdevice design and microfabrication. 


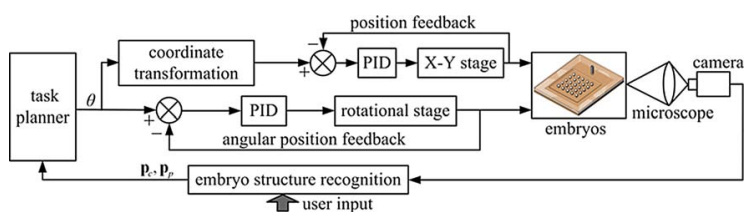

Fig. 8. 3-DOF closed-loop control for cell orientation.

\section{B. Visual Servo Control and Coordinate Transformation}

1) Feature Tracking: Feature tracking and visual servoing are only required when the system orients the first embryo within a batch. The system selects a portion of the initially userspecified polar body as a tracking target to provide the visual servo controller (see Fig. 7) with position feedback in the image coordinate frame. Considering rotational motions of the target image patch during embryo orientation, an sum-of-squared differences (SSD) algorithm with a translation-rotation-scaling (TRS) motion model [19] is employed for tracking the target image patch. The TRS motion model permits rotation-invariant visual tracking, and the algorithm is computationally efficient with real-time visual tracking capability. The SSD objective function is

$$
\operatorname{SSD}(\mathbf{u})=\sum_{\mathbf{x} \in \mathbf{T}}\left[I\left(f(\mathbf{x}, \mathbf{u}), t_{n}\right)-I\left(\mathbf{x}, t_{0}\right)\right]^{2}
$$

where $I\left(\mathbf{x}, t_{0}\right)$ is the intensity of point $\mathbf{x}$ in template image, $I\left(f(\mathbf{x}, \mathbf{u}), t_{n}\right)$ is the intensity of point $\mathbf{x}$ in the rectified image with motion parameters $\mathbf{u}$ at time $t_{n}$, and $f(\mathbf{x}, \mathbf{u})$ is the TRS motion model

$$
f(\mathbf{x}, \mathbf{u})=s\left[\begin{array}{cc}
\cos \theta & -\sin \theta \\
\sin \theta & \cos \theta
\end{array}\right] \mathbf{x}+\left[\begin{array}{c}
\Delta x \\
\Delta y
\end{array}\right]
$$

where $\mathbf{u}=[\Delta x, \Delta y, \theta, s]^{T}$ including translational parameters $[\Delta x, \Delta y]^{T}$, rotational parameter $\theta$, and scaling parameter $s$. By minimizing (1), $\mathbf{u}$ can be incrementally calculated. The increment $\Delta \mathbf{u}$ between two time instants is

$\Delta \mathbf{u}\left(t_{n}\right)=-\Sigma^{T}\left(\mathbf{M}_{\mathbf{0}}{ }^{T} \mathbf{M}_{\mathbf{0}}\right)^{-1} \mathbf{M}_{\mathbf{0}}{ }^{T}\left[I\left(f(\mathbf{x}, \mathbf{u}), t_{n}\right)-I\left(\mathbf{x}, t_{0}\right)\right]$

where $\mathbf{M}_{0}$ is an off-line computed constant matrix depending on the template image gradient and the TRS model, and $\Sigma$ is a matrix only determined by the TRS model. $\mathbf{M}_{0}$ is given by

$$
\mathbf{M}_{\mathbf{0}}=\left[\begin{array}{c}
\nabla_{\mathbf{x}} I\left(\mathbf{x}_{1}, t_{0}\right) \Gamma\left(\mathbf{x}_{1}\right) \\
\nabla_{\mathbf{x}} I\left(\mathbf{x}_{2}, t_{0}\right) \Gamma\left(\mathbf{x}_{2}\right) \\
\cdots \\
\nabla_{\mathbf{x}} I\left(\mathbf{x}_{N}, t_{0}\right) \Gamma\left(\mathbf{x}_{n}\right)
\end{array}\right]
$$

where $N$ is the number of pixels in the template image and $\nabla_{\mathbf{x}} I\left(\mathbf{x}_{i=1, \ldots, N}, t_{0}\right)$ is the spatial gradient of the template image at the location of $\mathbf{x}_{i}=\left[x_{i}, y_{i}\right]^{T}$, and

$$
\Gamma\left(\mathbf{x}_{i=1, \ldots, N}\right)=\left[\begin{array}{cccc}
1 & 0 & -y_{i} & x_{i} \\
0 & 1 & x_{i} & y_{i}
\end{array}\right] .
$$

Based on the TRS model, $\Sigma$ is derived as

$$
\Sigma=\left[\begin{array}{cccc}
\cos \theta / s & \sin \theta / s & 0 & 0 \\
-\sin \theta / s & \cos \theta / s & 0 & 0 \\
0 & 0 & 1 & 0 \\
0 & 0 & 0 & 1 / s
\end{array}\right]
$$

With a template of $31 \times 31$ pixels used in experiments, calculation of each $\Delta \mathbf{u}$ took $20.5 \mathrm{~ms}$. The tracking resolution was determined by tracking a stationary image patch of a mouse embryo and calculating the standard deviation.

2) Coordinate Transformation Calibration During Visual Servo Control: The system conducts coordinate transformation during visually servoed orientation control of the first cell within a batch (see Fig. 5, left column). Fig. 6 shows the 2-D coordinate frames of the rotational stage $\left(X_{r} O_{r} Y_{r}\right)$ and the $X-Y$ translational stage $\left(X_{t} O_{t} Y_{t}\right)$. The transformation from $X_{r} O_{r} Y_{r}$ to $X_{t} O_{t} Y_{t}$ is achieved by

$$
\left[\begin{array}{l}
x_{t} \\
y_{t}
\end{array}\right]=\left[\begin{array}{cc}
\cos \theta & -\sin \theta \\
\sin \theta & \cos \theta
\end{array}\right]\left[\begin{array}{l}
x_{r} \\
y_{r}
\end{array}\right]+\left[\begin{array}{l}
x_{t}^{r} \\
y_{t}^{r}
\end{array}\right]
$$

where $\left(x_{t}, y_{t}\right)^{T}$ is the target embryo coordinates in the $X-Y$ stage frame, $\left(x_{r}, y_{r}\right)^{T}$ is the target embryo coordinates in the rotational stage frame, $\theta$ is the angular position of the rotational stage, and $\left(x_{t}^{r}, y_{t}^{r}\right)^{T}$ is the coordinates of $O_{r}$ in the $X-Y$ stage frame. In (7), $\left(x_{r}, y_{r}\right)^{T}$ are unknowns to be calibrated during image-based visual servoing.

Upon the rotation of the first embryo, an image-based PID visual servo controller (see Fig. 7) is initiated to control the motorized $X-Y$ stage for keeping the visually tracked image patch inside the field of view and ultimately, bringing the cytoplasm center [see $\mathbf{p}_{c}$ in Fig. 4(b)] to the image center. The two angular positions $\theta_{1}, \theta_{2}$ and the corresponding coordinates $\left(x_{t 1}^{r}\right.$, $\left.y_{t 1}^{r}\right),\left(x_{t 2}^{r}, y_{t 2}^{r}\right)$ of the $X-Y$ stage before and after orientation are recorded and substituted into (7) to determine $\left(x_{r}, y_{r}\right)^{T}$.

\section{3-DOF Closed-Loop Position Control}

After the coordinate transformation calibration on the first embryo, other embryos within the same batch are oriented via closed-loop position control. Fig. 8 shows the system architecture of the closed-loop position control, where rotational control and $X-Y$ translational-position control are conducted simultaneously, constituting a 3-DOF closed-loop motion control configuration.

In Fig. 8, a task planner is responsible for determining the desired angular position $\theta$ of the rotational stage for embryo orientation based on the initially user-specified locations of the polar body and the cytoplasm centers $\left(\mathbf{p}_{c}\right.$ and $\left.\mathbf{p}_{p}\right)$. The calibrated coordinate transformation (see Fig. 8) maps the determined angular position $\theta$ in the rotational stage frame into desired Cartesian coordinates in the $X-Y$ stage frame for controlling the $X-Y$ stage to compensate for rotation-induced translational motions. PID controllers are used for regulating output motions of the rotational stage and the $X-Y$ stage.

\section{EXPERIMENTAL RESULTS}

The mouse embryos used were collected from ICR mice according to standard protocols approved by the Mount Sinai Hospital Animal Care Committee (Toronto). A $20 \times$ objective (NA 0.4 ) and differential interference contrast microscopy were used for embryo observation. The pixel size was calibrated to be 0.5 $\times 0.5 \mu \mathrm{m}$. 


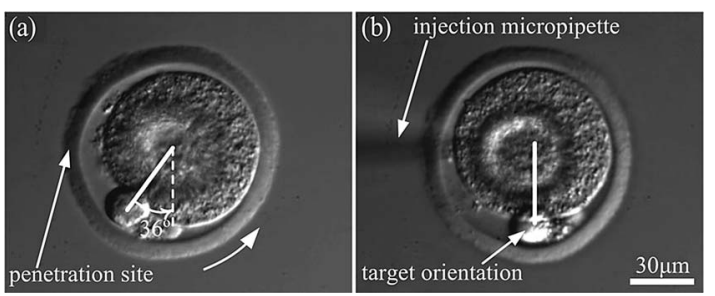

Fig. 9. Experimental snapshots of an embryo (a) before and (b) after automatic cell orientation. In (b), an injection micropipette tip lies at cytoplasm center before material deposition.
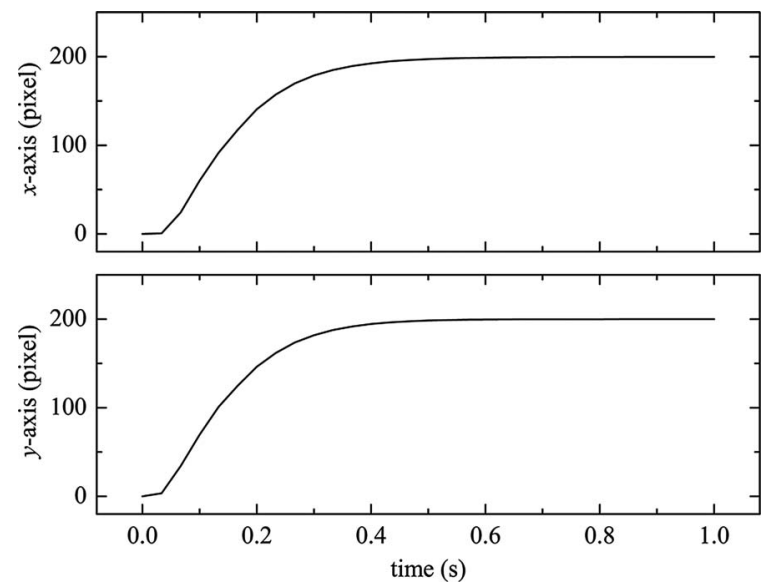

Fig. 10. Step response curves of the visual servo controller along $x$ - and $y$-axis in the image frame.

A set of 200 embryos ( 8 batches with a $5 \times 5$ cell holding device) at $3 \mathrm{~h}$ post-collection were tested. Of the 200 embryos, it was found that the polar body of 61 embryos (30.5\%) lied out of the initial focal plane and, therefore, required focal scanning for locating the polar body. The polar body of 19 embryos (9.5\%) appeared in the space of quadrant II (see Fig. 4), which required reorientation. The polar body of two embryos was found to be on the top or bottom of the embryo [along the vertical axis in Fig. 4(a)], where no reorientation was conducted. Fig. 9 shows experimental snapshots of an embryo before and after orientation control. After embryo orientation, each embryo was injected by the system [see Fig. 9(b)] to deliver foreign materials (e.g., protein molecules).

For each batch of cells, the image-based visual servo controller operates at $30 \mathrm{~Hz}$ for orienting the first embryo. The tracking resolution of the SSD algorithm was determined to be 0.2 pixel. Fig. 10 shows response curves of the image-based visual servo controller to a step input of 200 pixels along $x$ - and $y$-axis in the image frame. The settling time was $370 \mathrm{~ms}$, and the steady-state errors along both axes were zero.

The low bandwidth of the visual-servo controller limits the maximum rotational speed. The distance between the first embryo and the rotational axis $\left(R=\sqrt{x_{r}^{2}+y_{r}^{2}}\right.$ in Fig. 6) also determines the maximum rotational speed the system can afford. A large $R$ value results in a high rotation-induced translational

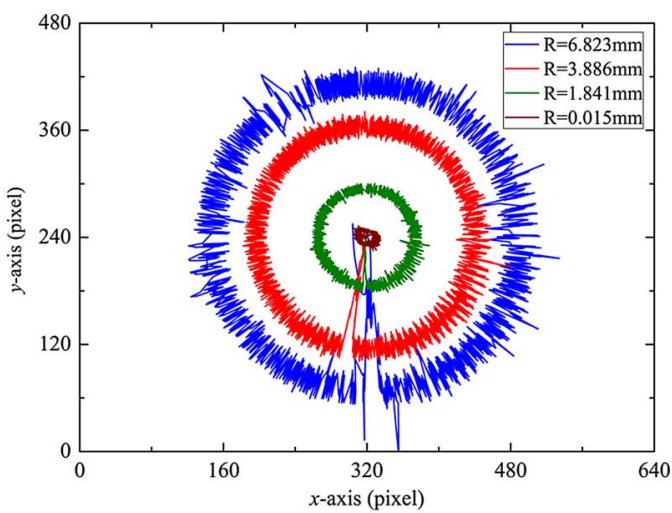

(a)

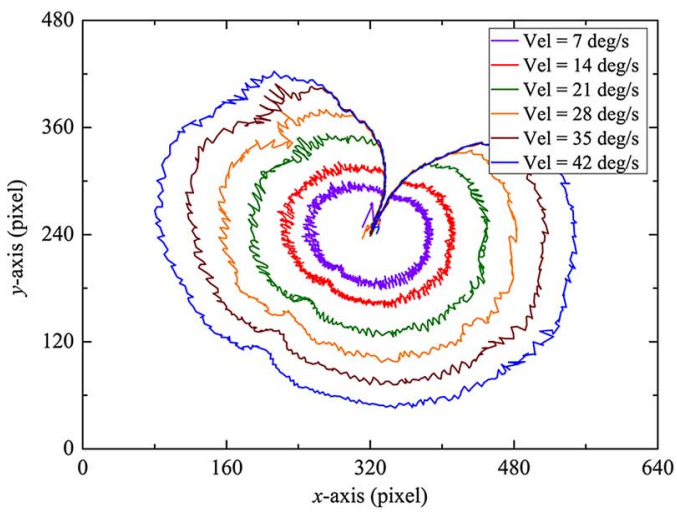

(b)

Fig. 11. (a) $X-Y$ stage trajectories for compensating for the rotation-induced translational motions, during visually servoed cell orientation for coordinate transformation calibration. Curves were collected on cells with different $R$ values $\left(R=\sqrt{x_{r}^{2}+y_{r}^{2}}\right.$ in Fig. 6). (b) $X-Y$ stage trajectories for compensating for the rotation-induced translational motions, during visually servoed cell orientation for coordinate transformation calibration. Curves were collected on the same cell $(R=0.1 \mathrm{~mm})$ but at different rotational speeds.

speed for the $X-Y$ stage to track, which further limits the maximum rotational speed.

Fig. 11(a) shows $X-Y$ stage trajectories for compensating for the rotation-induced translational motions and, therefore, keeping the embryo always within the field of view during orientation control of the first embryo in a batch for coordinate transformation calibration. Zigzags on the curves are from compensation motions of the $X-Y$ stage to keep the polar body in the field of view. The four curves correspond to cells with different $R$ values during visually servoed cell-orientation control at $7 \%$. Larger $R$ values generated larger zigzag motions and trajectories closer to image borders (i.e., higher chance for the visual servo controller to fail). For a given $R$ value, larger rotational speeds also resulted in $X-Y$ stage trajectories closer to image borders, as shown in Fig. 11(b) $(R=0.1 \mathrm{~mm})$. Visual servo control failed when the tracking target (i.e., the polar body) flew out of the field of view.

In experiments, the largest angle required is $90^{\circ}$ (see Fig. 4). The calibration process on the first embryo within a cell batch completed within $6 \mathrm{~s}$ at an orientation speed of $15^{\circ} / \mathrm{s}$. Then, the 3-DOF closed-loop position controller is capable of orienting 
the rest of the embryos within the same batch at $720 \%$ s (i.e., $<0.125$ s/cell).

The positioning accuracy of the 3-DOF closed-loop position control was also quantified. An embryo was orientated by a certain angle, and the $X-Y$ stage was correspondingly controlled to compensate for the rotation-induced translational motions calculated by the coordinate transformation and bring the cell back to its initial position inside the field of view. The translation accuracy is defined as the maximum deviation of a cell's position after orientation from its initial position inside the field of view. The rotation accuracy is defined as the maximum difference between the desired orientation angle and the one achieved by the rotational stage. From reorienting the 19 embryos that had their polar bodies present in the space of quadrant II (see Fig. 4), the translation accuracy was experimentally determined to be $1.3 \mu \mathrm{m}$; and the rotation accuracy was $0.24^{\circ}$. The rotation accuracy simply reflects the accuracy of the rotational stage. Error sources of the translation accuracy include the calibration error of coordinate transformation and positioning errors of the rotational stage and $X-Y$ stage.

\section{CONCLUSION}

This paper has presented hardware and control approaches for cell-orientation control under inverted microscopy. As an important component, a compact motorized rotational microscopy stage was developed. The polar body of mouse embryos was tracked in real time by an SSD visual tracking algorithm. An image-based visual servo controller was employed to keep the first target embryo in the field of view during orientation, when on-line calibration of coordinate transformation between the embryo holding device frame and cell positioning stage frame was also realized. The high-speed $(720 \%)$ cell orientation was achieved on the rest of the embryos in the same batch of cells via coordinate transformation and 3-DOF closed-loop position control (1-DOF rotational and 2-DOF translational). The technique enables automated, high-speed cell orientation for cell manipulation, and imaging tasks.

\section{REFERENCES}

[1] M. Savia and H. Koivo, "Contact micromanipulation-survey of strategies," IEEE/ASME Trans. Mechatronics, vol. 14, no. 4, pp. 504-514, Aug. 2009.

[2] S. Fatikow, T. Wich, H. Hulsen, T. Sievers, and M. Jahnisch, "Microrobot system for automatic nanohandling inside a scanning electron microscope," IEEE/ASME Trans. Mechatronics, vol. 12, no. 3, pp. 244-252, Jun. 2007.

[3] H. Xie and S. Regnier, "Development of a flexible robotic system for multiscale applications of micro/nanoscale manipulation and assembly," IEEE/ASME Trans. Mechatronics, pp. 1-11, 2010. DOI: 10.1109/TMECH.2010.2040483.

[4] Y. Zhang, B. Chen, X. Y. Liu, and Y. Sun, "Autonomous robotic pick-andplace of microobjects," IEEE Trans. Robot., vol. 26, no. 1, pp. 200-207, Feb. 2010.

[5] W. H. Wang, X. Y. Liu, D. Gelinas, B. Ciruna, and Y. Sun, "A fully automated robotic system for microinjection of zebrafish embryos," PLoS ONE, vol. 2, p.e862, 2007.

[6] F. Charriere, A. Marian, F. Montfort, J. Kuehn, T. Colomb, E. Cuche, P. Marquet, and C. Depeursinge, "Cell refractive index tomography by digital holographic microscopy," Opt. Lett., vol. 31, no. 2, pp. 178-180, 2006.
[7] N. M. Dragomir, X. M. Goh, C. L. Curl, L. M. D. Delbridge, and A. Roberts, "Quantitative polarized phase microscopy for birefringence imaging," Opt. Expr., vol. 15, pp. 17 690-17 6982007.

[8] Y. Kimura and R. Yanagimachi, "Intracytoplasmic sperm injection in the mouse," Biol. Reprod., vol. 52, no. 9, pp. 709-720, 1995.

[9] A. Nagy, M. Gertsenstein, K. Vintersten, and R. Behringer, Manipulating the Mouse Embryo - A Laboratory Manual, 3rd ed. New York: Cold Spring Harbor Laboratory Press, 2003.

[10] ProScan II inverted microscope motorized stage. (2010, Jul.) [Online]. Available: http://www.prior.com/productinfo_auto_proscan_h117.html

[11] K. Y. Kim, X. Y. Liu, Y. Zhang, and Y. Sun, "Nanonewton force-controlled manipulation of biological cells using a monolithic mems microgripper with two-axis force feedback," J. Micromech. Microeng., vol. 18, no. 5, pp. 055013-1-055013-8, 2008.

[12] K. Kobayashi, K. Kato, M. Saga, M. Yamane, C. Rothman, and S. Ogawa "Subzonal insemination of a single mouse spermatozoon with a personal computer-controlled micromanipulation system," Mol. Reprod. Dev., vol. 33, pp. 81-88, 1992.

[13] Y. Sun and B. J. Nelson, "Biological cell injection using an autonomous microrobotic system,” Int. J. Robot. Res., vol. 21, pp. 861-868, 2002.

[14] R. Kumar, A. Kapoor, and R. H. Taylor, "Preliminary experiments in robot/human cooperative microinjection," in Proc. IEEE Int. Conf. Intell. Robots Syst., 2003, pp. 3186-3191.

[15] L. S. Mattos, E. Grant, R. Thresher, and K. Kluckman, "Blastocyst microinjection automation," IEEE Trans. Info. Tech. Biomed., vol. 13, no. 5 , pp. 822-831, Sep. 2009.

[16] Y. Zhang, K. K. Tan, and S. Huang, "Vision-servo system for automated cell injection," IEEE Trans. Ind. Electron., vol. 56, no. 1, pp. 231-238, Jan. 2009.

[17] X. Y. Liu and Y. Sun, "Microfabricated glass devices for rapid single cell immobilization in mouse zygote microinjection," Biomed. Microdevices, vol. 11, pp. 1169-1174, 2009.

[18] X. Y. Liu and Y. Sun, "Visually servoed orientation control of biological cells in microrobotic cell manipulation," presented at the Int. Symp. Exp. Robot., Athens, Greece, Jul. 14-17, 2008.

[19] G. Hager and P. Belhumeur, "Efficient region tracking with parametric models of geometry and illumination," IEEE Trans. Pattern Anal. Mach. Intell., vol. 20, no. 10, pp. 1125-1139, Oct. 1998.

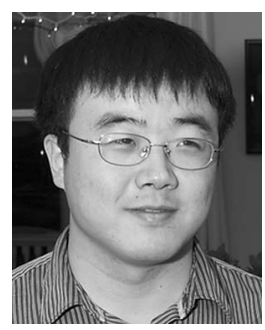

Xinyu Liu (S'06-M'10) received the B.Eng. and M.Eng. degrees in mechanical engineering from Harbin Institute of Technology, Harbin, China, in 2002 and 2004, respectively, and the Ph.D. degree in mechanical engineering from the University of Toronto, Toronto, ON, Canada, in 2009. His doctoral work primarily focused on microrobotics and MEMS-assisted manipulation and characterization of biological cells.

$\mathrm{He}$ is currently a Postdoctoral Fellow with the Whitesides Research Group at Harvard University, Cambridge, MA. His research interests include design and microfabrication of MEMS devices, micro and nanorobotic manipulation of bio and nanomaterials, low-cost diagnostic technologies for the developing world, and microfluidics for bioapplications. 


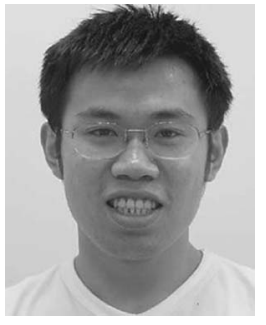

Zhe Lu (S'07-M'10) received the B.Eng. degree in control engineering from Harbin Institute of Technology, Harbin, China, in 2002, and the Ph.D. degree in mechanical engineering from the National University of Singapore, Singapore, in 2007.

$\mathrm{He}$ is currently a Postdoctoral Fellow in the Advanced Micro and Nanosystems Laboratory, Mechanical and Industrial Engineering Department, University of Toronto, Toronto, ON, Canada. His research interests include robotics, automation, and mechatronics.

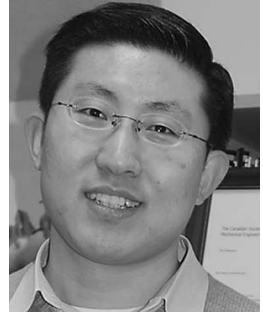

Yu Sun (S'01-M'03-SM'07) received the B.S. degree in electrical engineering from Dalian University of Technology, Dalian, China, in 1996, the M.S degree from the Institute of Automation, Chinese Academy of Sciences, Beijing, China, in 1999, the M.S. and Ph.D. degrees in electrical engineering and mechanical engineering from the University of Minnesota, Minneapolis, in 2001 and 2003, respectively.

$\mathrm{He}$ is currently an Associate Professor in the Mechanical and Industrial Engineering Department and is jointly appointed in the Institute of Biomaterials and Biomedical Engineering and Electrical and Computer Engineering Department, University of Toronto, Toronto, ON, Canada. He was a Research Scientist at the Swiss Federal Institute of Technology (ETH-Zürich) before joining the Faculty of Toronto in July 2004. He is a McLean Senior Fellow at the University of Toronto and the Canada Research Chair in Micro and Nano Engineering Systems. His research interests include design and fabrication of MEMS/NEMS devices; micro-nanorobotic manipulation under optical and electron microscopes; and manipulation and characterization of biological cells, biomolecules, and nanomaterials

Dr. Sun is on the Editorial Board of the IEEE TRANSACTIONS ON AUTOMATION SCIENCE AND ENGINEERING, IEEE/ASME TRANSACTIONS ON MECHATRONICS, and Micro and Nano Letters. 